\title{
A Distributed Algorithm for Bandwidth Allocation in Stable Ad Hoc Networks
}

\author{
Claude Chaudet ${ }^{1}$, Isabelle Guérin Lassous ${ }^{1}$, and Janez Žerovnik ${ }^{2,3 \star}$ \\ ${ }^{1}$ Inria Ares Team, Laboratoire Citi, Insa de Lyon, 21, avenue Jean Capelle, 69621 \\ Villeurbanne Cedex, France \\ Claude.Chaudet@insa-lyon.fr, Isabelle.Guerin-Lassous@inrialpes.fr \\ 2 University of Maribor, FS, Smetanova 17, 2000 Maribor, Slovenia \\ 3 Institute of Mathematics, Physics and Mechanics, Department of Theoretical \\ Computer Science, Jadranska 19, 1111 Ljubljana, Slovenia. \\ janez.zerovnik@uni-lj.si
}

\begin{abstract}
We propose a distributed algorithm for allocating bandwidth in stable ad hoc networks. After having discussed the problem of bandwidth allocation in such networks, we define a sequence of feasible solutions to this problem. This sequence has the property to be an increasing sequence in terms of overall used bandwidth. After a theoretical analysis of the sequence, we design a distributed algorithm based on this sequence. We test our algorithm by simulations on different topologies. Results obtained on geometric random graphs are presented here. We compare our solutions with the optimal solution in terms of global bandwidth allocation that presents the smallest standard deviation and with the the fairest solution regarding to max-min fairness. The simulations show that the global used bandwidth is less than $25 \%$ from optimality in the worst case and the standard deviation is the smallest of the three.
\end{abstract}

\section{Introduction}

Today, most of the existing wireless radio networks (GSM, WiFi) are infrastructure based networks. A fixed base station manages the transmissions in a certain geographic zone corresponding to its transmission range. Mobiles in this area need to be connected directly to this base station in order to communicate.

Mobile ad hoc networks are an evolution of these wireless networks in which no fixed infrastructure is needed. Mobiles communicate directly between each other, without the need for a base station. To enable these communications, any mobile should be able to perform routing for the others. The MANET (Mobile Ad hoc NETworks) working group has been created at the IETF in order to standardize a routing protocol for ad hoc networks. This working group has arrived in the final phase of its researches and is about to decide what the best effort routing protocol for these networks will be.

\footnotetext{
* Supported in part by the Ministry of Education, Science and Sport of Slovenia.
} 
In these networks, the radio medium is shared by all the mobiles. Many techniques are available to manage multiple access to the medium, from centralized or semi-centralized ones (time slot, frequency or spreading code allocation for example) to totally distributed ones (CSMA type for instance). Due to the infrastructure-less nature of ad hoc networks, the distributed medium access protocols seem more suited.

As most of the available wireless interface cards implement the IEEE 802.11 [1] standard, most of the actual ad hoc networks rely on it. This standard provides a totally distributed mode for the medium access part based on a CSMA / CA mechanism: the mobiles communicate on the same frequency and as long as one mobile emits, it prevents all of its neighbors from transmitting. Otherwise if two nearby mobiles emitted simultaneously on the same frequency, the two signals would interfere and there would be a high probability that none of them results in a successful transmission. As long as some bandwidth remains available, the MAC protocol manages to share quite efficiently the medium. As long as there is no congestion, mobiles can freely access the medium (in accordance with the 802.11 MAC protocol) and use whatever share of the resources they need. When the medium capacity is exceeded, the protocol behavior and the subsequent medium share become unpredictable.

Most of the proposed protocols for bandwidth management in ad hoc networks have an a posteriori approach, i.e. the bandwidth is managed only when congestion points appear. Protocols like INSIGNIA [2], SWAN [3] or HMP [4] do use such an approach. They provide notification, degradation or/and re-routing in order to react to the appearance of congestion. These approaches usually need additional communication between mobiles when the network already suffers from congestion. Therefore, returning to a stable situation can take some time. An alternative would be to manage the bandwidth a priori. If each mobile controls the use of its bandwidth rather than trying to use the whole radio medium, it will be able to prevent most of the congestion situations from appearing. It is difficult in such networks to avoid any congestion due to mobility: the control applied by one mobile in one configuration may not be adapted anymore in another configuration obtained by its mobility and may lead to the appearance of congestion. But an a priori solution may prevent from most of the congestion and may give efficient results in terms of bandwidth management as long as the network is quite stable with a low mobility.

In these networks, mechanisms like bandwidth reservation are essentially provided for constrained traffic, for example real-time flows. If the admission control is well suited to the characteristics of the network, reservations can prevent congestion from appearing. Best effort traffic is usually not limited by any mechanism and can thus easily overlap on the privileged traffics' bandwidth share, making the guarantees fragile. One possible solution to this problem is to allocate a constant amount of bandwidth for best effort traffic but this does neither take into account the resources required for the privileged traffic nor the topology of the network. Such solution often leads to a sub-optimal use of the network resources. An alternative is to allocate bandwidth to best effort traffic 
according to the properties of the network, i.e. the topology and the bandwidth available to each mobile. This assignment is done so that no saturation appears on any mobile. Finding such a solution while maximizing at the same time the overall used bandwidth in the network is equivalent to a fractional packing problem. Algorithms solving this problem are essentially sequential and difficult to adapt to a distributed setting. Moreover, such solutions maximize the total used bandwidth without guaranteeing any fairness among the mobiles. Not providing a minimum amount of bandwidth for each mobile in the network may lead to serious imbalance and to a bad use of the network.

In this article, we propose a distributed algorithm to allocate bandwidth to each mobile according to the topology of the network and the available bandwidth on each mobile for stable ad hoc networks. The algorithm guarantees a non null minimum bandwidth to each mobile. With this algorithm, each mobile computes the bandwidth it can use in order to avoid saturating its capacity or its neighbors'. With such an algorithm, congestion is less likely to appear in the network.

In Sect. 1, we give the used model for ad hoc networks and the bandwidth allocation problem. A simple bandwidth allocation is given in Sect. 2 From this allocation, we design a sequence of feasible allocation in Sect. [3] Each term of the sequence is a new bandwidth allocation that is more efficient in terms of global used bandwidth than the previous terms in the sequence. Different properties of the sequence are described in this section. From this sequence, we design a distributed algorithm that allocates bandwidth to each mobile of the network in Sect. 4 At each step of the algorithm, each mobile needs only to know the minimum remaining bandwidth and the maximum degree in its neighborhood. In Sect. 4.3 we present the results obtained by simulation on geometric random graphs. This work is on going and we evaluate in this article the quality of our allocation in terms of overall used bandwidth and of fairness among the mobiles. The obtained results should allow us to decide if our algorithm can be converted in an efficient bandwidth allocation protocol for ad hoc networks. In the conclusion, we discuss the points to solve for the protocol version and how to integrate mobility in our solution.

\section{The Model and the Problem}

We model our ad hoc network by a vertex weighted graph $G(V, E, b)$ where:

$-V=V(G)$ is the set of vertices of the graph. One vertex in the graph represents one mobile in the network;

$-E=E(G)$ is the set of edges of the graph. There is an edge between two vertices whenever the two corresponding mobiles are able to communicate, i.e. are in each other's transmission range;

$-b$ is a function which assigns positive real numbers to vertices, representing the capacity of the medium around each mobile.

A congestion appears whenever the capacity of the medium is exceeded in a certain region of the network. If we suppose that mobiles only share the medium 
with their direct neighbors, ensuring that for each mobile in the network, the sum of its used bandwidth and of the bandwidth used by its neighbors does not exceed this mobile's capacity will ensure that there is no congestion at all in the network. Formally, if, for each mobile $v$ in the network, we note $N[v]=\{v\} \cup\{u \mid u v \in E\}$ its closed neighborhood and $x(v)$ the amount of bandwidth that $v$ can use, the problem can be expressed as:

$$
\forall v \in V, \sum_{u \in N[v]} x(u) \leq b(v) .
$$

Maximizing at the same time the overall use of the network is equivalent to the following problem:

$$
\max \sum_{v \in V} x(v) \quad \text { s.t. } \forall v \in V, \sum_{u \in N[v]} x(u) \leq b(v) .
$$

This linear problem is known as a fractional packing problem. The problem can be solved by usual linear programming algorithms, and there are also faster approximation algorithms known (see 5] for details and for further references). These algorithms are sequential. A distributed algorithm for linear programming that obtains a $(1+\varepsilon)$-approximation in polylogarithmic number of communication rounds is given in [6]. Solutions to the fractional packing problem maximize the total bandwidth used in the network and give no guarantee on the minimum bandwidth for each mobile (i.e. the bandwidth may be null on some mobiles). This may have an impact on the good running of the network.

We propose a distributed algorithm that computes a set of values $\{x(v)\}_{v \in V}$ solution to the constraints (1). The algorithm is based on the topology of the network and the available bandwidth of each mobile. Before presenting the algorithm, we define the notation and formally evaluate the features of the solution in Sect. 2 and Sect. 3

\section{The Basic Lemma}

We will use the following notations:

$-N[v]$ is the closed neighborhood of the vertex $v$,

$-N(v)$ is the open neighborhood of the vertex $v(N(v)=N[v] \backslash\{v\})$,

$-d(v)$ is the degree of vertex $v$,

$-\mathbf{x}=(x(v), v \in V)$ is the vector of values we are trying to compute, i.e. the solution of (1), i.e. from a networking point of view, the allocated bandwidth to the mobiles,

$-\mathbf{b}=(b(v), v \in V(G))$ is the vector of the "capacities" of the vertices, i.e. from a networking point of view, the available bandwidth for each mobile,

$-\Delta_{1}(v)=\max _{u \in N[v]} d(u)$, i.e. the maximum degree over the closed neighborhood of $v$ ( $v$ included $)$, 
$-b_{1}(v)=\min _{u \in N[v]} b(u)$, i.e. from a networking point of view, the minimum bandwidth over the closed neighborhood of $v$ ( $v$ included).

Lemma 1. If for every node $v$ in the graph, $x(v)=\frac{b_{1}(v)}{\Delta_{1}(v)+1}$, then $\mathbf{x}=(x(v), v \in$ $V)$ is suitable to the constraints (1).

Proof. Recall from definitions of $\Delta_{1}$ and $b_{1}$ that $u \in N[v]$ implies that $d(v) \leq$ $\Delta_{1}(u)$ and $b(v) \geq b_{1}(u)$. Therefore,

$$
\begin{aligned}
\sum_{u \in N[v]} x(u) & \leq \sum_{u \in N[v]} \frac{b_{1}(u)}{\Delta_{1}(u)+1} \leq \sum_{u \in N[v]} \frac{b(v)}{d(v)+1} \leq \frac{d(v)+1}{d(v)+1} b(v) \\
& \leq b(v) .
\end{aligned}
$$

It means that $\mathrm{x}$ can be used as an initial solution to the constraints (1) and is a feasible bandwidth allocation.

\section{A Sequence of Feasible Vectors}

In Sect. 2. we have found a vector of values that respects the constraints defined by (1). In this section, we are going to show that if we iterate the process using the remaining bandwidth at each mobile after having computed $\mathbf{x}$, then we still have a solution to the problem and we increase the overall used bandwidth in the network.

\subsection{Sequence Definition}

We will consider the following sequences of vectors:

$-x^{(i)}(v)$ represents the allocated resources amount for the node $v$ at the $i^{t h}$ step of the sequence,

$-e^{(i)}(v)$ represents the remaining capacity of the node $v$ at the $i^{\text {th }}$ step considering what its neighbors have taken at this step.

These values are initialized as follows:

$$
\begin{gathered}
x^{(0)}(v)=\frac{b_{1}(v)}{\Delta_{1}(v)+1} . \\
e^{(0)}(v)=b(v)-\sum_{u \in N[v]} x^{(0)}(u) .
\end{gathered}
$$

Then the passage from step $i$ to step $i+1$ is done on the following way:

$$
\begin{gathered}
x^{(i+1)}(v)=x^{(i)}(v)+\frac{1}{\Delta_{1}(v)+1} \cdot \min _{u \in N[v]} e^{(i)}(u) . \\
e^{(i+1)}(v)=b(v)-\sum_{u \in N[v]} x^{(i+1)}(u) .
\end{gathered}
$$




\subsection{Sequence Properties}

Lemma 2. All the terms of this sequence respect the constraints defined by (1), i.e.

$$
\forall v \in V, \forall i \in \mathbb{N}, \sum_{u \in N[v]} x^{(i)}(u) \leq b(v) .
$$

Proof. We can write, $\forall v \in V, \forall i \in \mathbb{N}$,

$$
\begin{gathered}
\forall u \in N[v], \min _{w \in N[u]} e^{(i)}(w) \leq e^{(i)}(v) . \\
\Rightarrow \sum_{u \in N[v]}\left(\min _{w \in N[u]} e^{(i)}(w)\right) \leq|N[v]| \times e^{(i)}(v) .
\end{gathered}
$$

As $|N[v]|>0$, we can write:

$$
\frac{\sum_{u \in N[v]}\left(\min _{w \in N[u]} e^{(i)}(w)\right)}{|N[v]|} \leq e^{(i)}(v) .
$$

As $\forall u \in V, 1+\Delta_{1}(u) \geq 1+d(v)$ for any $v \in N[u]$ and $1+d(v)=|N[v]|$, then

$$
\sum_{u \in N[v]} \min _{w \in N[u]} e^{(i)}(w) \times \frac{1}{\Delta_{1}(u)+1} \leq e^{(i)}(v) .
$$

Therefore

$$
\begin{gathered}
\sum_{u \in N[v]}\left(x^{(i+1)}(u)-x^{(i)}(u)\right) \leq b(v)-\sum_{u \in N[v]} x^{(i)}(u) . \\
\Rightarrow \sum_{u \in N[v]} x^{(i+1)}(u) \leq b(v) .
\end{gathered}
$$

We have proved that $\forall v \in V, \forall i \in \mathbb{N}^{*}, \sum_{u \in N[v]} x^{(i)}(u) \leq b(v)$.

Thanks to Lemma 1, we know that

$$
\forall v \in V, \sum_{u \in N[v]} x^{(0)}(u) \leq b(v .)
$$

Therefore,

$$
\forall v \in V, \forall i \in \mathbb{N}, \sum_{u \in N[v]} x^{(i)}(u) \leq b(v) .
$$

It means that each element of the sequence is a solution to the constraints (11) and is a feasible bandwidth allocation. 
Lemma 3. If the vector $\mathbf{b}$ contains no zero then every allocated value is nonzero, i.e. $\forall v \in V, \forall i \in \mathbb{N}, x^{(i)}(v)>0$.

Proof. Straightforward with the definition of $x^{(0)}(v)$ and Lemma 2

It means that each term of the sequence corresponds to a bandwidth allocation where no mobile has a bandwidth equal to 0 .

Lemma 4. The sequence $\left(x^{(i)}(v)\right)_{i \in \mathbb{N}}$ is convergent.

Proof. We can write, using the definition of $e^{(i)}(v)$ and Lemma 2, that $\forall v \in$ $V, \forall i \in \mathbb{N}, e^{(i)}(v) \geq 0$. Therefore, the sequence $\left(x^{(i)}(v)\right)_{i \in \mathbb{N}}$ is monotone increasing. As a monotone increasing and bounded sequence always converges, and as $\left(x^{(i)}(v)\right)_{i \in \mathbb{N}}$ is bounded by $b(v),\left(x^{(i)}(v)\right)_{i \in \mathbb{N}}$ converges.

It means that each term of the sequence has an overall bandwidth greater than the previous terms in the sequence and that the sequence tends to a solution that has the maximum global bandwidth within this sequence.

Lemma 5. A node $v$ will reach a null remaining bandwidth at step $i\left(e^{(i)}(v)=0\right)$ if and only if this node has the minimum remaining bandwidth and the maximum degree among its neighbors at step $i-1$.

Proof. Assume that a node $v$ has a non null remaining bandwidth at step $i-1$ (i.e. $e^{(i-1)}(v)>0$ ) and does not have the maximum degree among its neighbors (i.e. $d(v)<\Delta_{1}(v)$ and $\exists z \in N(v)$ s.t.d $(z)=\Delta_{1}(v)$ ), by definition :

$$
\begin{aligned}
e^{(i)}(v) & =e^{(i-1)}(v)-\sum_{u \in N[v]} \frac{\min _{w \in N[u]} e^{(i-1)}(w)}{\Delta_{1}(u)+1} \\
& =e^{(i-1)}(v)-\left(\sum_{u \in N[v] \backslash\{z\}} \frac{\min _{w \in N[u]} e^{(i-1)}(w)}{\Delta_{1}(u)+1}\right)-\frac{\min _{w \in N[z]} e^{(i-1)}(w)}{\Delta_{1}(z)+1} .
\end{aligned}
$$

As $\forall u \in N[v], d(v) \leq \Delta_{1}(u)$ and as $\forall w \in N[u], \min _{w \in N[u]} e^{(i-1)}(w) \leq$ $e^{(i-1)}(v)$, then we have:

$$
\frac{\min _{w \in N[u]} e^{(i-1)}(w)}{\Delta_{1}(u)+1} \leq \frac{e^{(i-1)}(v)}{d(v)+1} .
$$

And as $\Delta_{1}(z) \geq d(z)>d(v)$ :

$$
\frac{\min _{w \in N[z]} e^{(i-1)}(w)}{\Delta_{1}(z)+1}<\frac{e^{(i-1)}(v)}{d(v)+1} .
$$

Injecting these two expressions in the previous one, we obtain:

$$
e^{(i)}(v)>e^{(i-1)}(v)-d(v) \cdot \frac{e^{(i-1)}(v)}{d(v)+1}-\frac{e^{(i-1)}(v)}{d(v)+1}>0 .
$$


Therefore, if a node has a non null bandwidth and does not have the maximum degree among its neighbors at a certain step, it will not reach its allocation limit at the next step. It is easy to show the same property for any node $v$ that has a non null remaining bandwidth at a given step and has a minimum remaining bandwidth greater than one of its neighbors'. Therefore a node can reach its limit at a given step only if it had the minimum remaining bandwidth and the maximum degree among its neighbors at the previous step.

Proving that a node with minimum remaining bandwidth and maximum degree among its neighbors at some step reaches its limit at the next step is straightforward from the definition of $x^{(i)}$.

This lemma characterizes the nodes that can reach a null remaining bandwidth. When they reach this state, their allocated bandwidth won't increase anymore in the next steps of the sequence and will remain constant. Note that the neighbors of such nodes will also reach the limit of their allocated bandwidth: they can't take more bandwidth in the next steps of the sequence otherwise they will saturate the capacity of the neighbor nodes that have a null remaining bandwidth.

\subsection{Quality of the Solution}

Lemma 6. Every node has at least a neighbor whose free bandwidth converges towards 0 :

$$
\forall v \in V, \exists u \in N[v] \text { s.t. } \lim _{i \rightarrow+\infty} e^{(i)}(u)=0
$$

Proof. As $\left(x^{(i)}(v)\right)_{i \in \mathbb{N}}$ converges, using the definition of the terms of the sequence: $x^{(i+1)}(v)=x^{(i)}(v)+\frac{1}{\Delta_{1}(v)+1} \min _{u \in N[v]} e^{(i)}(u)$, we can see that the number $x^{(i+1)}(v)-x^{(i)}(v)=\frac{1}{\Delta_{1}(v)+1} \min _{u \in N[v]} e^{(i)}(u)$ converges towards 0 . Therefore, $\min _{u \in N[v]} e^{(i)}(u)$ converges towards 0 .

Lemma 7. The sequence $\left(x^{(i+1)}(v)-x^{(i)}(v)\right)_{i \in \mathbb{N}}$ is decreasing.

Proof. As the sequence $\left(x^{(i)}(v)\right)_{i \in \mathbb{N}}$ is increasing, then the sequence $\left(e^{(i)}(v)\right)_{i \in \mathbb{N}}$ is decreasing. Then:

$$
\forall u \in N[v], e^{(i)}(u) \leq e^{(i-1)}(u)
$$

Therefore,

$$
\exists z \in N[v] \text { s.t. } \min _{u \in N[v]} e^{(i-1)}(u)=e^{(i-1)}(z) \geq e^{(i)}(z) \geq \min _{u \in N[v]} e^{(i)}(u) .
$$

And

$$
\left(x^{(i+1)}(v)-x^{(i)}(v)\right) \leq\left(x^{(i)}(v)-x^{(i-1)}(v)\right) .
$$


From now on, we will note $X(v)$ the limit of the sequence $\left(x^{(i)}(v)\right)_{i \in \mathbb{N}}, X$ the limit vector solution and $E(v)$ the remaining bandwidth at node $v$ with the solution $X$.

Lemma 8. $X$ is Pareto-efficient.

Proof. We consider the order $\leq$ where $\leq$ is the natural order on $\mathbb{R}^{N}$, i.e. $x \leq y$ iff $x_{i} \leq y_{i}, \forall i \in[1, N]$. A solution is Pareto-efficient [7] if it is maximum in the sense of $\leq$.

Assume we have a vector $S$ that respects the constraints (11) and so that $S>$ $X$ according to the order previously defined. This means that there exists a node $v$ so that $S(v)>X(v)$. According Lemma 6 there exists a node $u \in N[v]$ that has reached its bandwidth allocation limit. This means that $\sum_{w \in N[u]} X(w)=b(u)$. As $S(v)>X(v)$ and as $S$ respects the constraints (1), necessarily there exists a node $z \in N[u] \backslash\{v\}$ such that $S(z)<X(z)$. Therefore we can not have $S>X$.

\subsection{Convergence Speed}

Not much can be said on this algorithm convergence speed. The speed is highly dependent on the graph topology and on the bandwidths available at each node.

On one hand, for a regular graph with uniform weights $(\forall v \in V, b(v)=b)$, the algorithm finds the optimal solution for the fractional packing problem at the first step (i.e. $\forall v \in V, x^{(0)}(v)=\frac{1}{1+\Delta} b$ and $e^{(0)}(v)=0$ ).

On the other hand, we can find networks configurations that result in a sequence that converges with an infinite number of steps. For example, consider a chain of three nodes. Nodes of index 1 and 3 can communicate with node of index 2 but not between themselves. Assume that $b(1)=b(3)=2$ and $b(2)=3$. After one step, the remaining bandwidth at each node is exactly divided by 3 (2/3 for nodes 1 and 3 and 1 for node 2$)$ and the bandwidth keep the same ratios between each other. Therefore, all further steps will lead to a configuration in which the ratio between the bandwidths is the same as initially and hence the number of steps to converge is, in this configuration, infinite.

Now, if we consider the same configuration with $b(1)=b(3)=2 \cdot b(2)$, the algorithm converges in a single step.

As we will show in Sect. 4.3. simulations give good results in the number steps of our algorithm needs to converge to a value near the limit of the sequence, whatever the network may be.

\section{A Distributed Algorithm for Bandwidth Allocation}

\subsection{Base Algorithm}

The following distributed algorithm Algorithm 1 is based on the sequence presented in Sect. 3. It computes an increasing sequence and each element of the sequence is a solution to the constraints (1). The algorithm locally computes in each vertex the sequence $\left(x^{(i)}(v)\right)_{i \in \mathbb{N}}$ stored in the variable $X$. At the end 
of the algorithm, $X$ gives the bandwidth allocated at node $v$. The remaining bandwidth $\left(e^{(i)}(v)\right)_{i \in \mathbb{N}}$ is stored in each vertex in the variable $E$. The information that needs to be gathered for the computation consists, at each step, in the values $\left(x^{(i)}(u), e^{(i)}(u)\right)_{i \in \mathbb{N}}$ for each neighbor and the degree of each neighbor. These informations need to be broadcasted in two steps as the calculation of one sequence requires the updated value of the other sequence.

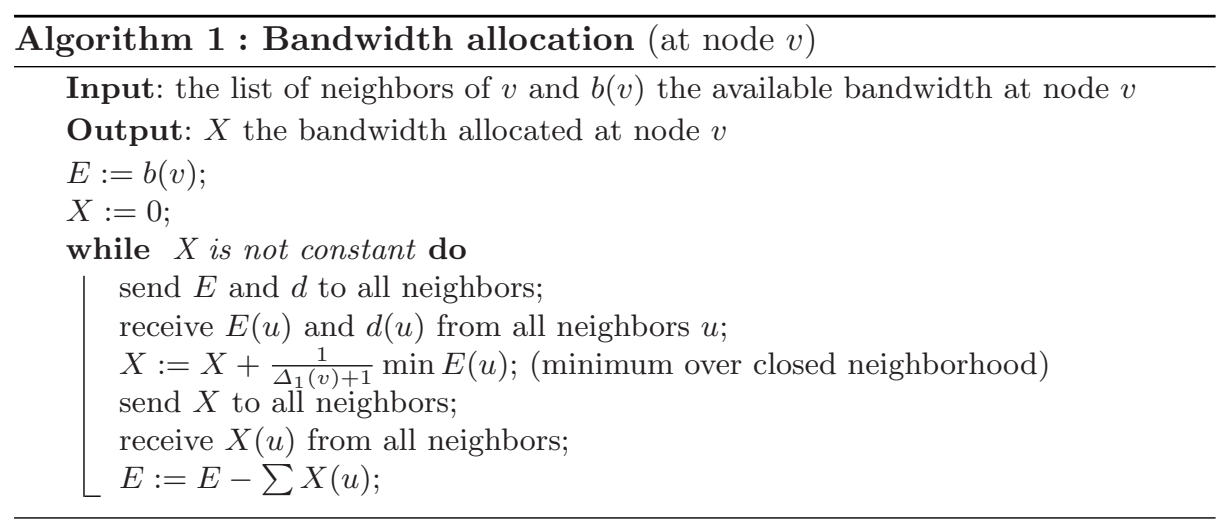

\subsection{Remarks on This Algorithm}

- By Lemma 4, the algorithm clearly converges to a feasible solution $X$. Each term of the sequence $X^{(i)}$, represents a feasible solution for constraints (1) and gives the bandwidth that can be used by each mobile in the network. Each term $X^{(i)}$ gives an overall bandwidth greater than with the previous terms, but note that $X$ is not always optimal in terms of global bandwidth as some examples presented in the next section will show.

- By Lemma 2, we know that the bandwidth allocated to the mobile with Algorithm 1 does not exceed the capacity of the network. This allocation is fair in the sense that no mobile overlaps on its neighbors resources amount. Moreover by Lemma 3 all mobiles do get some resources.

- By Lemma 6, we know that there is no "space left" in the resources once the algorithm is finished. That does not mean that all mobiles may use the total capacity of their wireless cards, but that means that each mobile has a neighbor in its closed neighborhood that has no space left. Thus, if a mobile wants to use more bandwidth than the one allocated then a congestion point will appear in its neighborhood because it will exceed the capacity of its neighbor that has no capacity remaining.

- By Lemma 7, we know that the difference between two consecutive values of $X$ is decreasing. According to the quality of the solution we want to obtain, we can consider, at each step of the algorithm, that this difference becomes small enough to accept this solution and to not go further with the algorithm. 
Therefore, instead of achieving $X$ constant, we can stop the algorithm when the difference between two consecutive values of $X$ is smaller than a given threshold. We discuss the impact of this threshold in the next section.

- We also designed and tested a simple optimized version. In this second version, the nodes also transmit the minimum bandwidth in their neighborhood. If this value is 0 , then neighbors will know this node will not be able to take anymore bandwidth. Then, they don't have to take it into account in the max degree calculation in the next step. They nevertheless still need to be considered when looking at the minimum bandwidth in the neighborhood. Depending on the network topology, this optimized version may have a strong impact on the convergence with some configurations. We will compare the convergence speed of these two versions in Sect. 4.3.

\subsection{Implementation Results of This Algorithm}

Algorithm 1 has been implemented in $\mathrm{C}++$. It has been tested on different kinds of configurations, like complete graphs, chains, rings, meshes and geometric random graphs. Due to space limitations, we will only present here the results obtained on geometric random graphs, as these graphs are in our opinion the most accurate representation of ad hoc networks. Other results can be found in the extended version of this article [8]. 50 runs of each test have been carried out on different sizes of the configurations and we give the average results.

The geometric random graphs we used to test our algorithm are generated by considering 100 nodes put in a $1000 \times 1000$ square and by increasing the communication range of each node. The communication area ranges from 130 to 200 meters. When the communication range increases, the average degree of the nodes also increases. Each node is given a random capacity (available bandwidth b) between 50 and 150 .

To determine the quality of our algorithm, we compare our solution with a solution to the fractional packing problem and with the most fair solution respecting the constraints (1) regarding a max-min fairness criteria.

A solution to the fractional packing problem may be computed by classical linear programming using the simplex algorithm. This results in a solution respecting the constraints (11) that maximizes the overall used bandwidth but that is not necessarily fair, as it may assign 0 bandwidth to some nodes. As the optimal solution to the linear programming maximization problem defined by (11) is not unique, we needed to find the "most fair" of these solutions. We choose to use standard deviation between the allocated bandwidths as the fairness criteria. To find the most fair optimal solution according to this criteria, we need to minimize the standard deviation between the allocated bandwidths subject to the constraints defined by (10) to which we add another constraint: the sum of all the bandwidths has to be equal to the objective function value computed by linear programming. This quadratic problem was solved using OOQP [9] and the results are presented below. As the time complexity of quadratic programming relatively large and the computation is sequential, the properties of the solutions 
can only be used as benchmarks for comparison and evaluation of the results of our algorithms.

We have also computed the most fair solution regarding to the max-min fairness criteria. This solution is often considered as the fairest allocation, as noted in 1011 . As a quick overview, in the max-min fairest solution to a problem, no bandwidth can be increased without decreasing another bandwidth that is already lower than the first one. Our solution is not the max-min fairest solution (it is easy to find a counterexample). We compare our solution with the max-min optimal solution.
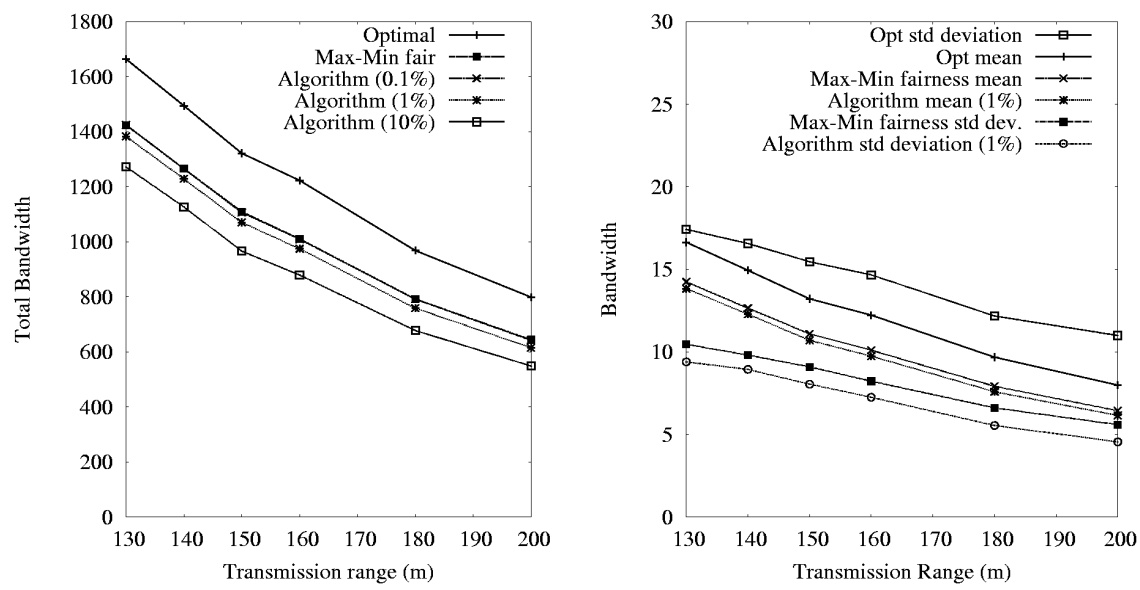

Fig. 1. Global bandwidth and statistics on the obtained solution on geometric graphs

The quality of the solution in terms of global bandwidth use is represented by the left plot of Fig. 1. First, we can notice that the global bandwidth decreases as the communication range increases. This fact can be explained by the increase of the constraints of the system due to the increase of the average degree of the nodes and by the constant number of nodes. The obtained bandwidth with our algorithm is between $18 \%$ and $25 \%$ smaller than the optimal one. The overall bandwidth with the max-min solution is slightly greater than with our solution. The increase in global bandwidth is around $9 \%$ for 200 meters when the threshold switches from $1 \%$ to $0.1 \%$. On the other hand, this increase is much smaller with sparse networks (it is around 5\% with 130 meters).

To give an indication on the fairness of the solution, we can compare the mean value and the standard deviation of the obtained solution with the two other solutions. These statistics, shown on the right plot of Fig. 1, show that our algorithm is quite fair with this configuration. The values allocated to the nodes range from around 4 to 24 in average for a communication range of 130 meters and from around 1 to 11 in average for a communication range of 200 meters. Our algorithm is fairer on dense networks than on sparse ones. As for 
the other configurations, the standard deviation is around more than two times greater with optimal solution than with our solution. The difference between the max-min standard deviation and ours is more visible with these networks and is about $10 \%$.

Lastly, we compare the convergence times of the initial algorithm and the modified algorithm as described in Sect. 4.2. Each result has been obtained with different stopping thresholds (as discussed in Sect. 4.2). Three values of this threshold have been considered: $10 \%, 1 \%$ and $0.1 \%$ (it means that for a threshold of $1 \%$ for instance, the algorithm stops as soon as two consecutive values of $X$ differ for less than $1 \%$ ).

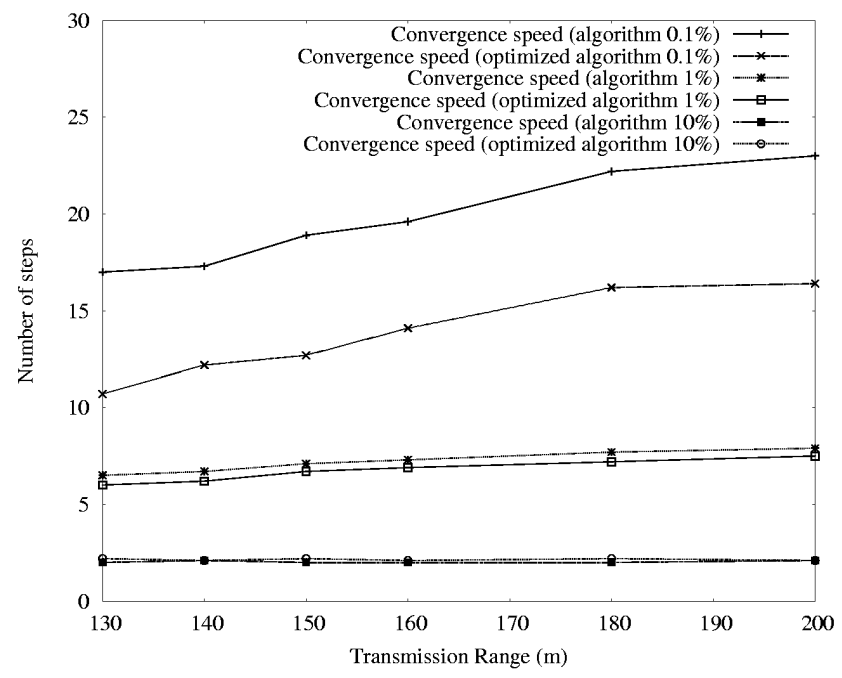

Fig. 2. Number of steps of Algorithm 1 on geometric graphs

Figure 2 shows that the convergence speed depends on the degree of the nodes, especially when the threshold is $0.1 \%$. The maximum convergence speed is obtained with the initial algorithm with a threshold of $0.1 \%$ and is around 22 steps for the worst situation with a communication range of 200 meters. In this configuration, the convergence is reduced to 16 steps with the modified version. As for the other configurations, the speeding up increases with the refining of the threshold.

The results (accessible in [8]) obtained for chains, rings and meshes and complete graphs show that our algorithm is not far from the optimal in regular configurations (about 10\% below the optimal global bandwidth). Moreover, the standard deviation is generally rather smaller than the standard deviation of the most fair optimal solution as well as the one of the max-min most fair solution.

By comparing these results, we can say that the number of nodes in the networks has a very limited impact on the quality of our solution (mean value, stan- 
dard deviation and convergence speed) as long as the degrees remain constant. On the other hand, the degree is an important parameter and our algorithms perform better on dense graphs regarding fairness than on sparse ones and better on sparse graphs than on dense graphs regarding total bandwidth allocation and convergence speed.

\section{Conclusion and Future Work}

The results achieved by this algorithm are promising and encourage us to convert it into a protocol in order to test its stability and to determine its influence on congestion points appearance.

Nevertheless, this conversion will not be straightforward. Mobility is also a key issue. Ad hoc networks are supposed to be mobile networks and the protocol will have to take into account topology changes. One solution is for each mobile to check the feasibility of the allocation considering appearing links regarding the constraints (1). If one link violates one of the constraints, the involved mobiles on the link may then share the minimum initial allocated bandwidth between these two nodes. If this solution quickly backtracks to a feasible solution, it may lead to an allocation less fair than the proposed solution of this article. Another alternative is that a mobile suffering an over-allocation implies its neighbors in the return to a stable solution. This kind of mechanism would probably lead to a fairer allocation but it could take some time before stabilizing. Our future work is to investigate such solutions.

\section{References}

1. IEEE Standard for Information Technology Telecommunications and Information Exchange between Systems: Local and Metropolitan Area Network - Specific Requirements - Part 11: Wireless LAN Medium Access Control (MAC) and Physical Layer (PHY) Specifications. (1997)

2. Lee, S.B., Ahn, G.S., Zhang, X., Campbell, A.T.: Insignia: An ip-based quality of service framework for mobile ad hoc networks. Journal on Parallel and Distributed Computing 60 (2000)

3. Ahn, G.S., Campbell, A.T., Veres, A., Sun, L.H.: SWAN: Service differentiation in stateless wireless ad hoc networks. In: IEEE INFOCOM' 2002, New York, USA (2002)

4. Lee, S.B., Campbell, A.T.: HMP: Hotspot mitigation Protocol for Mobile Ad Hoc Networks. In: 11th IEEE/IFIP International Workshop on Quality of Service, Monterey, Canada (2003)

5. Plotkin, S.A., Schmoys, D.B., Tardos, E.: Fast approximation algorithms for fractional packing and covering problems. Mathematics Of Operations Research 20 (1995) 257-301

6. Bartal, Y., Byers, J.W., Raz, D.: Global optimization using local information with applications to flow control. In: 38th IEEE Symp. on Foundations of Computer Science. (1997) 303-312

7. Feldman, A.M.: Welfare Economics and Social Choice Theory. Kluwer, Boston (1980) 
8. Chaudet, C., Guérin Lassous, I., Zerovnik, J.: A distributed algorithm for bandwidth allocation in stable ad hoc networks. Technical Report 4827, INRIA (2003)

9. Gertz, M., Wright, S.: Object-oriented software for quadratic programming. Technical Report ANL/MCS-P891-1000, Argonne National Laboratory, Mathematics and Computer Science Division (2001)

10. Bonald, T., Massoulié, L.: Impact of Fairness on Internet Performance. In: Proceedings of SIGMETRICS, Cambridge, MA, USA (2001)

11. Bertsekas, D., Gallager, R.: Data Networks. Prentice Hall (1987) 